\title{
Proteasome disability syndrome
}

INSERM

\section{Source}

INSERM. (1999). Orphanet: an online rare disease and orphan drug data base.

Proteasome disability syndrome. ORPHA:324977

Proteasome disability syndrome describes a group of autosomal recessively inherited autoinflammatory disorders characterized by lipodystrophy and skin eruptions. The disorders belong ing to this group include Nakajo-Nishimura syndrome (NNS), JMP syndrome and CANDLE syndrome (see these terms) and all are caused by mutations in the PSMB8 gene (6p21.3). 